\title{
ANOTACIONES PARA UNA REFLEXIÓN SOBRE LA EDUCACIÓN SUPERIOR EN COLOMBIA
}

\author{
RAMÓN FAYAD, PH.D. ${ }^{1}$ \\ 1 Profesor Titular, Facultad de Ciencias Naturales y Matemáticas, Universidad del Rosario, Bogotá, Colombia
}

\begin{abstract}
Resumen
La universidad es una institución que ha sufrido grandes transformaciones a lo largo de su historia. A pesar de la autonomía con la que realiza sus actividades y organiza sus estructuras, no se ha mantenido al margen de las demandas de la sociedad, sobre todo en la formación de profesionales. Hace dos siglos, en Europa y en Norteamérica, la universidad se convirtió en el espacio en el que profesores y estudiantes comenzaron a realizar investigación científica, crear conocimiento, contextualizarlo y preservar la cultura. La realidad actual indica que el conocimiento se ha convertido en un patrimonio que sin duda ha contribuido al desarrollo y a mejorar las condiciones de vida de las sociedades. En este trabajo se ilustra cómo el sistema de educación superior colombiano es muy débil, pues si bien cuenta con un gran número de instituciones de educación superior, la escasa oferta de programas de doctorado y el número muy bajo de profesores que sean investigadores activos se refleja en la poca producción de conocimiento que se genera en sus universidades, salvo en contadas excepciones. Se sugieren acciones inmediatas para que se replantee el sistema de educación superior y se articule a un sistema de ciencia y tecnología. La situación actual muestra que de no iniciarse acciones concretas que estimulen y financien adecuadamente la investigación científica, Colombia seguirá rezagada en el contexto científico mundial, sin universidades de investigación y condenada al subdesarrollo económico y social.
\end{abstract}

Palabras clave: educación superior, ciencia, tecnología, conocimiento

\section{ANNOTATIONS FOR A REFLECTION ON THE HIGHER EDUCATION IN COLOMBIA}

\begin{abstract}
The university as an institution has undergone great transformations throughout its history. Despite the autonomy with which it carries its activities and organizes its structures, it has not remained at the margins of the demands of society, mostly in the formation of professionals. Two centuries ago, in Europe and North America, the university became the space in which professors and students began to develop scientific research, to create knowledge, to put it in context, and to preserve the culture. The current reality indicates that knowledge has become a patrimony that, without a doubt, has contributed to the development and improvement of the life conditions of the societies. In this work it is shown that the Colombian system of higher education is too weak, that in spite of the great number of higher education institutions, the offerings of doctoral programs is scarce and the low number of active researchers is reflected in the short list of production of knowledge in the universities, except in a few examples. Some concrete actions are suggested so that the higher education system can be articulated
\end{abstract}

* Correspondencia: Ramón Fayad ramon.fayad@gmail.com, ramon.fayad@urosario.edu.co. Dirección Postal: Facultad de Ciencias Naturales y Matemáticas, Universidad del Rosario, Carrera 24 No. 63C-69, Bogotá, D.C. Colombia.

Recibido: Mayo 12 de 2010 Aceptado: Junio 23 de 2010. 
to a scientific and technological system. The present situation indicates that if not immediate actions are taken in order to stimulate and to adequately finance scientific research, Colombia will continue to be behind the scientific world context, without research universities and it will be condemned to the economic and social underdevelopment.

Keywords: Higher education, science, technology, knowledge

\section{Resumo}

A universidade é uma instituição que tem sofrido grandes transformações ao longo de sua história. Apesar da autonomia com que desenvolve as suas actividades e organiza as suas estruturas, não se manteve distante das demandas da sociedade, especialmente na formação profissional. Dois séculos atrás, na Europa e América do Norte, a universidade tornou-se o espaço em que professores e alunos começaram a realizar pesquisas científicas, criar conhecimento, contexto e preservação cultural. A situação atual indica que o conhecimento tornou-se um património que, sem dúvida, contribuiu para o desenvolvimento e melhorar as condições de vida das sociedades. Este trabalho ilustra como o sistema de educação superior na Colômbia é muito fraco, porque embora tenha um grande número de instituições de ensino superior, um suprimento insuficiente de programas de doutoramento e do número muito reduzido de professores que são pesquisadores ativos é refletida na baixa produção de conhecimento gerado nas universidades, com poucas exceções. As ações imediatas são sugeridos para reconsiderar o sistema de ensino superior e ser organizado a ciência e tecnologia. A situação atual mostra que sem ações específicas lançadas para incentivar e financiar a investigação científica de forma adequada, a Colômbia fica para tras no contexto científico global, sem universidades de investigação e apontando ao subdesenvolvimento económico e social.

Palavras chave: educação superior, ciencia, tecnologia, conhecimento

\section{Los orígenes}

Desde su creación en la Edad Media la universidad ha diversificado las ramas del saber que se cultivan en su interior, ha establecido estructuras que organicen sus funciones y ha replanteado su relación con los demás sectores de la sociedad.

Cuando en tiempos medievales se requirió mano de obra calificada, a la universidad, que se dedicaba a recrear el pasado, se le encargó la elaboración de planes de estudio que capacitaran a las personas para el desempeño de las pocas profesiones que se exigían. Adicionalmente a la esencia de la autonomía en lo académico, lo administrativo, lo económico y lo organizacional, se le autorizó para que ejerciera una función que tendría un gran efecto público: conferir títulos académicos reconocidos en los principales ámbitos de la sociedad como eran la carrera eclesiástica, el campo jurídico y la práctica de la medicina. Se contaba con cuatro facultades: teología, leyes, medicina y artes. Las tres primeras se encargaron de establecer los requisitos propios de la profesión respectiva, mientras que las artes se referían a los demás saberes que no tenían por qué estar relacionados con el desempeño de los oficios de la época.

A comienzos del siglo XVI se puso fin a las denominadas pretensiones universalistas de la universidad, quizás como consecuencia del Cisma de Occidente y de los cambios de concepción tanto sobre las diversas clasificaciones del saber como de algunas áreas del conocimiento (1). Se empezaron a consolidar las bases de la ciencia moderna, surgió la demanda por la formación de profesionales en áreas como la ingeniería y aparecieron los primeros elementos de los estados-naciones. Se inició la fundación de universidades europeas, se replantearon las estructuras administrativas y se crearon más facultades, adicionales a las cuatro tradicionales. Es de resaltar que en muchos casos lo público de la universidad, particularmente la vinculación de profesores y la oferta de programas, 
comenzó a depender, más que de una política estatal, de la orientación ideológica de quienes ostentaran el poder gubernamental (2).

En contraste con la universidad medieval, hacia 1550 la labor de las universidades se dedicó más a cultivar el saber contemporáneo. Algunas tendencias optaron por apoyar decididamente a las universidades que ofrecieran programas estrictamente profesionales pero en otros casos se decidió privilegiar actividades como el cultivo de las ciencias humanas y la pura reflexión intelectual. Un caso interesante es el de la Universidad de Praga, en la que, ya en esos tiempos, dadas las diversificaciones del quehacer profesional, las facultades se organizaron con personas que compartían áreas comunes y se les asignó la función central de actualizar permanentemente los programas académicos correspondientes, definir los saberes y habilidades que debían poseer los estudiantes para obtener un título y anticiparse a las necesidades de profesionales que demandaría la sociedad aun en futuros lejanos. Aquí nació el concepto de estructura curricular de un programa universitario.

Las universidades existentes irían transformándose y adecuarían su actividad a las propuestas de quien tuviera el control del Estado. El caso más notable fue el de Francia, en donde las universidades orientaron el quehacer académico hacia la formación puramente profesional, de manera que los egresados ingresaran a la fuerza laboral de los entes estatales. Esta concepción napoleónica de universidad dio origen al bien conocido fortalecimiento de las escuelas profesionales o vocacionales que posteriormente se crearon en otros países.

A comienzos del siglo XIX, en Alemania se inició una transformación en el sistema educativo. Wilhelm von Humboldt sentó las bases para que, exclusivamente con recursos del Estado, se creara una universidad en Berlín. Se definieron como misiones institucionales de la universidad la docencia y la investigación científica y humanística. Se fortalecieron los planes de estudio de las profesiones con bases de ciencias y humanidades y se consagró el ejercicio de la libertad de cátedra. En palabras de Humboldt "... el profesor de universidad no es un maestro, ni el estudiante un educando, sino alguien que investiga por sí mismo, guiado y orientado por el profesor". Con la vinculación de investigadores como profesores y con estudiantes que culminaban sus programas con aportes nuevos al conocimiento, se dio origen a los programas doctorales stricto sensu y a lo que hoy se denomina "universidad de investigación".

Si bien el modelo alemán se expandió en el continente europeo, adaptado a las particularidades de cada nación, en los Estados Unidos este modelo produjo un impacto mayor. Los estudiosos del tema han llamado la atención sobre este punto pues, para esa época, el sistema norteamericano de educación superior se hallaba inmerso en un libre mercado del que no escapaban universidades tan antiguas y reconocidas como Harvard, Princeton y Yale. El modelo trascendió y lo adaptaron las universidades estaduales, inspiradas inicialmente en la Morrill Land Grant Act poco después de la mitad del siglo XIX, en donde se competía por recursos y alumnos y la investigación era generalmente en artes mecánicas y agrícolas. Se inició una decidida política estatal estadounidense de financiar con significativos recursos públicos la investigación básica y aplicada. Adicionalmente a estos recursos se sumaron, como continúa ocurriendo en el presente, las donaciones en dinero y en especie que los particulares hacían a las universidades para apoyar la investigación científica y la creación de becas en estudios avanzados.

\section{El modelo latinoamericano}

En Latinoamérica influyó más la concepción napoleónica. En las universidades creadas por el Estado aparecieron facultades de corte técnico, cuyos nombres se asignaban de acuerdo con la respectiva profesión, denominación que aún se mantiene en varios países de la región. A finales del siglo XIX en las universidades aparecieron los departamentos y cada uno definía y administraba el currículo propio de su área del saber, de manera que se optó por una formación estrictamente monodisciplinar.

A pesar de la mencionada influencia napoleónica, en algunas universidades los profesores asumieron la investigación científica como parte de su quehacer. Varios profesionales dedicaron la mayor parte del tiempo al trabajo académico y se ocuparon de la formación de estudiantes. Así, en Colombia, en 1935, se legalizó la profesión de profesor universitario. Además de la docencia, apareció formalmente, y por primera vez, la investigación como una de las funciones de los profesores. Con contadas excepciones, la misión de la universidad en esta región se limitó a formar profesionales en las áreas tradicionales de medicina, leyes, agronomía e ingeniería. La unidad encargada de ad- 
ministrar un programa en un área del conocimiento se denominó, arbitrariamente, facultad o departamento y adoptó el nombre del programa que administraba. Se encontraba indistintamente, por ejemplo, Facultad de Ingeniería Civil, Departamento de Ingeniería Civil y Carrera de Ingeniería Civil. La denominación de facultad, departamento y programa curricular, utilizadas como sinónimos, fue un equívoco que se mantuvo hasta bien entrado el siglo XX y, como se mencionó, aún se conserva en algunas instituciones de la región.

En la década 1960-1970 las comunidades académicas no encontraban espacios de desarrollo intelectual en el esquema de las universidades profesionalizantes y el modelo napoleónico se vería agotado. En Colombia se dieron importantes transformaciones en las estructuras universitarias que permitieron replantear el quehacer universitario; los campos del saber disciplinar y profesional se redistribuyeron de una manera más acorde con el área del conocimiento que aglutinara tanto a los saberes como a los profesores. Varias unidades que eran facultades pasaron a ser departamentos de las nuevas facultades. Se crearon las Facultades de Ciencias Naturales, Ciencias Humanas, y Artes, que, con la oferta de cursos y otras actividades, le dieron un soporte más completo e integral a las profesiones. Ahora serían las facultades y no los departamentos las que administrarían los programas, abandonando así la formación monodisciplinar y dándoles un carácter más universal. Se inició, además, la creación y puesta en marcha de programas formales en disciplinas como la física, la química y la biología.

Unas décadas antes, en Argentina, Brasil, Chile y México se había adoptado una posición intermedia entre la universidad napoleónica y el modelo alemán. La investigación era una actividad reconocida que desarrollaban grupos importantes de profesores junto con la docencia y se había iniciado una serie de acciones concretas para institucionalizarla, no solamente como ejercicio intelectual sino como un quehacer estratégico para impulsar el desarrollo de los países, en el sentido más amplio del término. Es posible que algunas de las reformas iniciadas en la Francia del "mayo del 68" hayan influido en los replanteamientos de la educación superior en los países de la región, pero no es menos cierto que desde antes se habían creado entes estatales para delinear políticas y apoyar financieramente la investigación científica. Tal es el caso de $\mathrm{CNpQ}$ en Brasil (1951), Conicet en Argentina (1958), Conicit en
Venezuela (1958), Cinvestav en México (1961), Conicyt en Chile (1968) y Colciencias en Colombia (1968).

Las condiciones sociopolíticas mundiales de mediados del siglo pasado, a las que se agregó la escasa financiación de las universidades públicas en Latinoamérica, llevó a una serie de conflictos que intentaron, afortunadamente sin éxito, desprestigiarlas y presentarlas como focos de alteración del orden establecido. Un efecto notable fue la aparición de gran cantidad de instituciones privadas, muy heterogéneas y en no pocos casos de calidad cuestionable, que se autodenominaron universidades. A la vez, algunos gobernantes regionales habían creado una variedad de instituciones denominadas también universidades, sin infraestructura mínima de lo que corresponde a una entidad de educación superior. Varios sectores, basándose en que la educación es un servicio público que puede prestarse por particulares, lo acomodaron a un servicio al público y transformaron la libertad de cátedra en la libertad de empresa, como lo habían hecho algunos empresarios mercantilistas cien años antes en los Estados Unidos. Por fortuna para Colombia, unas instituciones han tratado de consolidarse $\mathrm{y}$ ofrecen varios programas de calidad que son una alternativa para los nacionales que no han tenido acceso, por diversas razones, a las universidades de mayor desarrollo.

\section{Dos normas generales en los últimos 30 años}

En Colombia, con el propósito de poner orden a la proliferación dispersa, por qué no anárquica, de instituciones y programas, se expidió la ley 80 de 1980. Se dio explícito reconocimiento a la actividad investigativa como una componente del quehacer de la universidad, se definieron los programas de maestría y doctorado de manera que sus planes de estudio estuvieran articulados en torno a la investigación, y se fijó un monto, en términos de porcentaje de su presupuesto, que debían destinar las universidades públicas para actividades de investigación. Se definió la connotación universalmente aceptada de los títulos de doctor, en los que el eje de la formación es la investigación, de manera que para obtenerlo se debía culminar con un aporte original a la creación de conocimiento y plasmarse en una tesis pública que tenía que ser avalada por pares. La eliminación del término "doctor" en los diplomas de pregrado todavía produce gran malestar en profesionales que egresan de las áreas del derecho y la medicina, por no mencionar otras profesiones, en una sociedad que 
está acostumbrada a llamar "doctor" al egresado de cualquier programa y de cualquier nivel.

Otros decretos expedidos durante ese año y los inmediatamente siguientes, relacionados con el estatuto de profesores de las universidades públicas, incorporaron la productividad académica, en la que una de sus modalidades sustanciales era la publicación de artículos científicos y la creación original en humanidades, artes y filosofía. Es de anotar que, a pesar de que se incorporó la investigación como actividad propia de los profesores, las normas se expidieron utilizando los términos "docente universitario", y no "profesor universitario".

Esta extensa ley, que llegó incluso a fijar por norma la estructura de las universidades públicas, las formas de designar sus órganos de gobierno y nombrar los cargos directivos, perdió la oportunidad de llamar a las cosas por su nombre. Les dio características de universidad a las instituciones que estuvieran en capacidad de ofrecer un mínimo de tres programas, por tanto que contaran con por lo menos tres facultades. Muchas instituciones bastante débiles adelantaron rápidamente todo tipo de acciones burocráticas y con actos meramente administrativos crearon los programas y las facultades exigidas por la norma, convirtiéndose, casi por autodeterminación, en universidades.

A pesar de la ley, continuó la hipertrofia de instituciones y programas; los tipos de formación (técnico, tecnólogo y universitario) siguieron diferenciándose por modalidades, mas no por niveles de formación, y su duración en periodos académicos era incierto. Como no se hizo suficiente énfasis en la importancia de cada uno de estos tipos de educación para el funcionamiento y transformación de los diferentes actores de la sociedad, ésta no los asumió como formaciones diferentes sino que los categorizó, dándole incluso calificativos peyorativos a la modalidad de técnico.

Con el fin de poner orden al nuevo desorden se expidió la ley 30 de 1992. En ésta se reconoció la autonomía universitaria, y se derogaron las normas que imponían rigidez a las estructuras de las universidades públicas. Apareció, y está aun en el ambiente, una tensión entre la autonomía y la función de inspección y vigilancia que la Carta Política le asigna al Ejecutivo pues algunos la consideran como una intromisión indebida en una institución cuya actividad es la búsqueda de la verdad sin concesiones. La autonomía no ha sido motivo de grandes conflictos en el sector, excepto para quienes consideran que el punto central de la autonomía radica en que las autoridades universitarias deben elegirse por voto directo de profesores, estudiantes y personal administrativo, y para aquellos que la esgrimen cuando, dizque en defensa de lo público, acuden a la intimidación y a la violencia para apropiarse privadamente de los espacios. Sin embargo, sería interesante que los estudiosos adelantaran comparaciones entre lo que ocurre en Colombia con la calidad de las instituciones y de los programas que se ofrecen con lo que acontece en otros países, en los que la intervención estatal es nula y en los que la educación está totalmente intervenida. En otras palabras, analizar si el sistema educativo por sí solo, y en todos sus niveles, está en capacidad de autorregular la calidad de sus funciones.

En el marco de esta ley, cuyo contexto se socializó ampliamente en el país (3), los programas se definieron en términos de una ordenación por niveles, mas no por modalidades, con estimados de tiempo en periodos académicos en los cuales se espera que los saberes y habilidades deben aprehenderse de acuerdo con la aproximación al tema de estudio y a la problemática que enfrentara el egresado en su desempeño en el campo laboral. La investigación pasó a ser un elemento constitutivo de la calidad de los programas universitarios, incluso en los de pregrado, y se reafirmó la relación de los programas de posgrado con la investigación; ésta, además, debía ser el elemento distintivo de las universidades en el conjunto de las Instituciones de Educación Superior (IES) y se reafirmó la importancia de las ciencias básicas, humanas, sociales y las artes en los planes de estudio de las formaciones profesionales, tal y como se había hecho en la universidad humboldtiana 190 años antes. Sin embargo, el legislador cometió un error conceptual importante al incluir los posdoctorados como programas de formación avanzada. Éstos, en todas las regiones del mundo, no son programas; son actividades de alto nivel que se realizan después del doctorado pero no conducen a título.

En la ley, con el propósito de desarrollar el sistema de educación superior, delinear políticas, asegurar la calidad y mantener informada a la ciudadanía, se crearon organismos tan importantes como el Consejo Nacional de Acreditación (CNA), el Sistema Nacional de Información de la Educación Superior (SNIES) y el Consejo de Educación Superior Universitario (CESU); en este último tienen cabida académicos y represen- 
tantes tanto de los entes estatales del sector educativo como de otros sectores de la sociedad. Además, se le dio piso legal al Sistema de Universidades del Estado (SUE), tema que se había planteado por los académicos desde hacía varias décadas en Colombia. Hasta ahora, por tratarse de instancias relativamente nuevas en el país, se ha requerido de algunos ajustes en su funcionamiento y la sociedad ha ido reconociendo la conveniencia de contar con éstos. El significado y los procesos de acreditación, por ejemplo, tardaron algunos años antes de que las instituciones, sus directivas y los demás miembros de la comunidad académica comprendieran su significado y los procedimientos a seguir en el caso de someter a acreditación tanto programas como instituciones. Un paso siguiente tiene que ser el de articular el quehacer de las IES con las políticas de los Planes de Ciencia y Tecnología que existen formalmente en Colombia desde 1983 y que ahora es un mandato constitucional en el que se fijan algunos principios que relacionan la educación con el desarrollo de la ciencia y la tecnología.

En el momento de sancionar la ley se perdió, o al menos se pospuso una vez más, la posibilidad de llamar a algunas cosas por su nombre. Como se mencionó, se establecieron las características de las instituciones que debían ser universidades, en las cuales la investigación científica, humanística, artística y filosófica sería su elemento distintivo; pero el legislador decidió consignar que serían también universidades aquellas que, en el momento de expedirse la ley, se denominaban como tales. Algunas instituciones que por su inadecuada infraestructura física, por la debilidad en el número y calidad de sus profesores, por el bajo nivel de formación que impartían y por la ausencia total de actividades investigativas, condujeron a que el lenguaje popular las llamara "de garaje". Como se denominaban universidades, la norma las legitimó. Aún casi dos décadas después de sancionada la ley varias de estas instituciones no han hecho esfuerzos significativos por mejorar; lamentablemente la situación es irreversible, aún a mediano plazo, pues nadie parece estar dispuesto a pagar el costo político que representa ponerlas en su debido lugar e impedir que lleguen a jugar no solo con el dinero sino con las ilusiones de los estudiantes. Una cosa es que una institución anuncie en los diferentes medios de comunicación los programas que ofrece, como ocurre con las instituciones serias, y otra que, como las "de garaje", promocionen la compra del futuro promisorio y asegurado si se ingresa en ellas.
Es posible extenderse en la descripción de la normatividad vigente en el país, casi toda impulsada por los sectores académicos, y que no difieren mayormente de las que rigen en otros países de la región. Pero el asunto no es de normas sino de personas. Los analistas coinciden en afirmar que las universidades son refractarias a las transformaciones, que no gratuitamente en Latinoamérica la universidad se ha calificado como la "institución más conservadora", tal vez por la tradición cultural de mantener sus orientaciones desde su origen en la época colonial y por sus limitados desarrollos en el campo científico, social y tecnológico. Así las cosas, ante la ausencia de políticas estatales y universitarias claras, en algunas instituciones la investigación sigue considerándose como algo marginal y exótico; se opta más bien por mantener programas académicos exhaustivos en información sin formar para la actividad creativa; se prepara a las personas más para la ocupación de empleos que, en la época actual, rápidamente pierden vigencia a menos que el profesional esté perfeccionando su formación de pregrado, preferiblemente con programas de posgrado, en los que desarrolle la capacidad de creación e innovación.

\section{Datos sobre el sistema de educación superior}

Según datos del Ministerio de Educación Nacional (4) en el país hay 283 IES, de las cuales 80 son universidades. Solo $4,1 \%$ de los profesores tiene formación doctoral y del total apenas $30 \%$ son de tiempo completo. Estos datos ameritan comentarios, así sean muy generales. Ante todo, el número de las llamadas universidades es exageradamente alto si se analiza en términos de lo que no solo la ley sino las comunidades académicas entienden por universidad. Si nos atenemos a los conceptos universalmente aceptados, en Colombia, con grandes esfuerzos, no encontraríamos más de 12 universidades. Aquí se ven las consecuencias de la laxitud de las normas al hacer concesiones. La incoherencia es evidente, como ya se mencionó, pues acepta que se llamen universidades aún aquellas instituciones que no reúnen los requisitos de la definición. Podría decirse entonces, en una acomodación libre, que se trata de una contradictio in terminis,

Los datos de SNIES son bastante completos y muestran la localización de instituciones, programas y profesores. $\mathrm{Si}$ alguien, ingenuamente, distribuyera el número de profesores, su dedicación y su nivel de formación entre el número de instituciones, se encontraría con una situación, ahora sí, lamentable. El mayor número de 
profesores de tiempo completo, con doctorado y maestría, que son además investigadores activos, están vinculados a pocas universidades públicas, principalmente a las de nivel nacional, y a muy escasas privadas. Son precisamente estas universidades las que tienen mayor número de proyectos aprobados en Colciencias, las que ofrecen el mayor número de los programas de posgrado, particularmente de los 90 de doctorado, y en los que hay mayor número de grupos de investigación que tienen reconocimiento de Colciencias y están en las más altas categorías (5). Por ejemplo, de los 4.075 grupos que reunían los requisitos de la convocatoria de 10 de junio de 2010, en donde están incluidos los de instituciones que no son de educación superior, los resultados muestran que hay 6 universidades con más de cien grupos: Universidad Nacional de Colombia (637), Universidad de Antioquia (225), Universidad del Valle (169), Universidad Javeriana (162), Universidad de los Andes (153), Universidad Pedagógica y Tecnológica de Colombia (112). Siguen la Universidad Industrial de Santander (97), la Universidad Tecnológica de Pereira (88), la Universidad del Cauca (84) y la Universidad Pedagógica Nacional (59). La distribución geográfica es muy desequilibrada pues se tienen regiones en donde la educación superior es inexistente mientras hay zonas en las que se encuentra el mayor porcentaje de instituciones de calidad. Por tanto, es necesario replantear las estrategias que permitan contar con un sistema que cubra con calidad a todas las regiones del país pues, de lo contrario, el sistema no es nacional.

De nuevo, las cifras deben mirarse con cuidado; sin embargo, sin esperar el análisis de los expertos, puede llamarse la atención sobre varios aspectos. Es en las universidades mencionadas donde, como se anotó, se encuentran las mayores fortalezas de los indicadores que se han venido utilizando para estudiar la calidad de la educación superior en el país. Debe precisarse que nunca se ha pretendido ordenarlas de mayor a menor calidad; simplemente se están estudiando estas correlaciones con el propósito de contar con elementos que permitan elaborar el estado del arte para articular de manera armónica el Sistema de Educación Superior al Sistema de Ciencia y Tecnología en el contexto de las realidades actuales. Sorprende que las IES que reclaman tener más estudiantes que cualquiera de las universidades mencionadas no cuenten con un solo grupo de investigación clasificado. Es más llamativo que en varios casos el número de grupos clasificados de una institución no corresponda, por ser demasiado alto, con la actividad por la que se les conoce: los niveles de pregrado de los programas que ofrece, su actividad básicamente docente, con pocos profesores de tiempo completo y sin planta suficiente con título de posgrado.

La definición de grupos y la necesidad de tener información sobre ellos son temas de interés general para toda la sociedad. Se reflejan las áreas en las cuales se está trabajando en investigación en el país, cuál es su impacto en la creación de conocimiento y en la producción artística, permiten identificar no solamente a las personas que la están adelantando sino a qué IES u otro tipo de organización están vinculadas. Por otra parte, debe disponerse de datos confiables que faciliten la elaboración de políticas que orienten las acciones a seguir desde Colciencias para coadyuvar al fortalecimiento de las instituciones en las que la investigación sea parte de la cotidianidad, tanto como lo es la docencia. Lamentablemente hay grupos en los que se da la asociación entre investigadores que no han trabajado conjuntamente pues el propósito es clasificarse en categorías altas, recibir reconocimientos en sus respectivas instituciones y tener acceso a mayor asignación financiera en el momento de formular proyectos, que es lo que ocurre con el efecto Mateo. Por supuesto que la colaboración estrecha entre investigadores e instituciones es absolutamente necesaria; en un país como Colombia, es más conveniente para todos los sectores aunar las fortalezas que competir con las debilidades. Es crucial que la iniciativa de Colciencias se respalde con datos fieles suministrados por las IES y por los investigadores; si las cifras con las que alimentan las plataformas no son cuidadosas, los resultados de las convocatorias se distorsionan y el buen propósito se puede desvirtuar. Si se miran solamente los números, en los dos últimos años la situación ha progresado, al menos cuantitativamente; pero si no se es claro en el suministro de información, la existencia de un número tan grande de investigadores activos y su asociación, a veces ficticia, en grupos a los que no están vinculados estudiantes que en poco tiempo se conviertan en investigadores autónomos y continúen la formación de otros, podría dar la impresión de que se cuenta con universidades completas, incluso con universidades de investigación, que no es por ahora el caso en el país. Los documentos que resumen los resultados de un encuentro celebrado en marzo de 2008, al que asistieron el Ministerio de Educación Nacional, el CESU, el CNA y la Comisión Nacional para la Calidad de la Educación Superior (CONACES) presentan aspectos profundos sobre el papel de la investigación en las 
diferentes modalidades de programas de pregrado y posgrado que se ofrecen en Colombia (6). Se revela que la actividad investigativa, que califican de "precaria" aún comparándola con algunos países de la región, no ha mejorado sustancialmente en lo cualitativo a pesar del optimismo que pueden despertar las cifras.

Si se vuelve a los datos del Ministerio de Educación Nacional de 2010 (4), se encuentra que del total de estudiantes matriculados en todos los niveles que ofrecen las IES, es decir en los 9.626 programas, $1,3 \%$ están en maestrías y apenas $0,1 \%$ en programas doctorales. Del total de estudiantes graduados en el sistema en 2009 , solamente $0,1 \%$ obtuvo el título de doctor. Se concluye que la oferta de programas doctorales es muy baja, lo mismo que el número de personas que los adelantan y se gradúan, mientras que el total de programas que se ofrecen es extremadamente alto. El respaldo, más bien retórico del Estado a la formación avanzada, sumado a la oposición de los sectores más retrógrados, premodernos y menos preparados de las instituciones, de algunos profesores que prefieren desempeñarse con contratos vitalicios en ambientes de la universidad que se describió de comienzos del siglo $\mathrm{XX}$, muestra que en el país se está muy lejos de contar con universidades de investigación. Sin embargo, hay universidades que en los 10 últimos años han tomado iniciativas, han vinculado a números muy significativos de profesores con doctorado y le han apostado a la investigación y creación de conocimiento como uno de los pilares de su actividad, lo que sin duda contribuirá a consolidación de comunidades académicas, beneficiará a la docencia de calidad y a la proyección de ese conocimiento a los demás sectores de la sociedad.

En 2007 la cobertura del sistema colombiano de educación superior fue $32,9 \%$, apenas 2,2 puntos por debajo del promedio de América Latina y el Caribe. Tanto la tasa de deserción, que fue $12,4 \%$ en ese año, como la larga permanencia de los estudiantes en muchos programas y en varias instituciones debe ser motivo de análisis. Es necesario continuar con la recopilación de información que permita establecer correlaciones entre el número de estudiantes matriculados en un programa, la tasa de retención, pero, sobre todo, la tasa de graduación de las cohortes en los tiempos estipulados. Si se aumenta la cobertura a expensas del hacinamiento de estudiantes en las aulas y laboratorios, si no se incrementan los aportes para la adecuación de espacios y las dotaciones que permitan el desarrollo de pedagogías interactivas, y si no se aumenta la tasa de graduación, sin hacer concesiones en la calidad de los programas, el concepto de cobertura pierde significado. Si bien la deserción, que es un tema muy complejo y generalizado al menos en Latinoamérica, ha encontrado algunas explicaciones tanto por la juventud $e$ inmadurez de quienes ingresan a los programas sin una vocación definida, como en términos de necesidades económicas que los conducen a ocupar la mayoría del tiempo en actividades remuneradas, es de interés, como se está haciendo en algunas instituciones, correlacionar el rendimiento en asignaturas o actividades complementarias y las que forman parte del núcleo básico del programa respectivo. Es frecuente que haya alto rendimiento en estas últimas y muy bajo en las primeras, lo que conlleva frustraciones y abandono del programa. Es innegable que las instituciones, a través de los programas de bienestar, adelantan acciones de seguimiento y apoyo a los estudiantes; al menos y a pesar de que las cifras de deserción siguen siendo altas, el promedio en los últimos cinco años no ha aumentado. En este punto, como en todo lo que atañe al sistema, debe adelantarse urgentemente una política estatal que trate de resolver este problema que tiene un costo social muy grande.

\section{Ranking de las universidades}

No se trata de controvertir la intención de clasificar a las universidades ni de poner en tela de juicio los indicadores que se utilizan para ordenarlas por su visibilidad, que se ha asociado con calidad. Como lo han anotado los analistas, "los indicadores suministran una medida de la producción académica en la red y de la interactividad que dicha producción genera con múltiples actores dentro y fuera de las respectivas regiones y países (academia, sector productivo, organizaciones sociales de diverso tipo, públicas y privadas). La documentación publicable en los portales web de las instituciones de educación superior incluye producción académica formal e informal, que refleja las actividades de profesores e investigadores" (7).

El "Ranking Web de las universidades del mundo" producido por el laboratorio de Cibermetría del Consejo Superior de Investigaciones Científicas de España (7), cubre 18.000 universidades de todo el mundo y ofrece información acerca de las primeras 8.000 en cuanto a calidad. Los resultados muestran que las primeras 20 universidades del mundo son de los Estados Unidos. Dentro de las primeras 500 universidades del mundo, 200 son de los Estados Unidos y Canadá; 
223 son europeas y clasifican 14 latinoamericanas (Brasil, Argentina, México y Chile) que están en los países de la región que a comienzos del siglo XX no se mantuvieron rígidos frente al modelo napoleónico de universidad. Entre las primeras 1.000, Latinoamérica ubica 44 (de nuevo Brasil, México, Argentina y Chile) y ninguna colombiana. Entre las 100 primeras universidades latinoamericanas, Brasil clasifica 43, México 18, Argentina, 11, Chile 8, Colombia 7. Los analistas de estudios de competencia y mercado en educación superior en el contexto de la globalización, ya en 2004 habían anticipado estos resultados (8), pues, sin saber que se harían, tenían claro que la relación de desarrollo de las instituciones no es simétrica. Hay universidades cuyo prestigio descansa en la investigación, en su papel como escuelas mundiales de posgrado $y$, por qué no decirlo, en el reconocimiento de su "marca", como es el caso de muchas universidades de los Estados Unidos y del Reino Unido. Otro caso es el de las universidades de gran prestigio en su propio país pero de poco peso relativo a nivel mundial, como ocurre en Japón y en Australia. En los últimos segmentos siempre estarán las instituciones sin o con escasa investigación, con actividad dentro de un ámbito que no traspasa fronteras y cuyos referentes son las otras instituciones locales. Éste es, sin duda, el caso de las IES colombianas como puede predecirse al menos con el indicador de las publicaciones científicas, y el porcentaje del Producto Interno Bruto (PIB) que invierte Colombia en investigación y desarrollo (I\&D) para no mencionar los otros indicadores en los que, como se mencionó, no debe aspirarse, al menos por ahora, a comparaciones que decepcionen. Un tema tan obvio en otras latitudes como el que los profesores tengan título de doctor, es una tarea a largo plazo que, como política en Colombia, apenas algunas universidades la iniciaron hace pocos años.

Si se comparan los indicadores que caracterizan al sistema de educación superior colombiano con los de los llamados países industrializados, incluso con los de algunos países de la región, es posible hacer un análisis serio que desnude las debilidades. Un poco peor es el resultado si se tienen en la cuenta indicadores tales como el número de profesores con doctorado $e$ investigadores activos que hay en la institución, cuántos de ellos han sido galardonados con el Premio Nobel o la Medalla Fields, la cantidad e impacto de sus publicaciones y patentes, el número de investigadores por habitante, las inversiones como porcentaje del $\mathrm{PIB}$ en I\&D y el número de graduados en programas avanzados cuyo eje central es la investigación. No es muy difícil predecir la posición que ocuparía Colombia en un contexto universal.

Los datos del CINDA (9) muestran que en 2004 en revistas indexadas se publicaron en Brasil 17.324 artículos, de los cuales 4.874 se produjeron en la Universidad de Sao Paulo; la Universidad de Buenos Aires produjo 15.888; la Universidad Nacional Autónoma de México 2.637; la Universidad Nacional de Colombia 220; la Universidad de Antioquia 150; la Universidad de los Andes 74 y la Universidad Industrial de Santander 53. De los datos más generales se concluye que Colombia aporta el $2 \%$ de la producción científica de todos los países iberoamericanos. Los aportes de éstos contribuyen con el $4 \%$ de la producción científica mundial. Los datos del Ministerio de Educación Nacional (4) reportan que en ese año en Brasil se graduaron 6.492 doctores, en México 893, en Chile 104, en Colombia 43 y en Estados Unidos 58.747. La meta propuesta por la llamada "comisión de sabios", era contar con cerca de 40.000 doctores en Colombia en 2010 y se tienen algo menos de 5.000. Sí ha habido esfuerzos pero no son suficientes; en los últimos 10 años se han formado unos 700 doctores, pero este número debería ser la meta anual mínima para las próximas dos décadas. Es cierto que se empezó con rezagos pero no es posible sustraer del tema las condiciones socioculturales del país y las marcadas influencias napoleónicas. Valga recordar que los doctorados se iniciaron en Alemania en la segunda década del siglo XIX y en Colombia en la penúltima del siglo XX. Poner en marcha un programa doctoral exige, además de intenciones, grandes inversiones, sobre todo si es en áreas que requieran equipos robustos para adelantar experimentación. Como se ha insistido, la estrategia tiene que partir de políticas estatales que faciliten la captación de recursos suficientes y sostenibles. Es innegable que los datos comparativos de los diez últimos años muestran un avance cuantitativo en varios indicadores del sistema de educación superior y del sistema de ciencia y tecnología. Además, la transformación de Colciencias en Departamento Administrativo es una buena señal, pero debe garantizársele un presupuesto adecuado y sostenible para que pueda cumplir su función, como se hizo en su momento con el SENA. Una posibilidad, además de aumentar significativamente el porcentaje del PIB que se invierte en I\&D para que alcance cifras del orden del $1 \%$, es destinar también una cifra importante de las regalías, por lo menos el $15 \%$, para apoyar proyectos de Ciencia, Tecnología e Innovación. 


\section{Comentarios finales}

La brecha entre países como Colombia y el resto del mundo en ciencia y tecnología irá aumentando de no adoptarse estrategias que pongan en marcha acciones para elevar el nivel de la educación, desde la básica hasta el doctorado. En escuelas, colegios e IES debe estimularse la actitud investigativa de los estudiantes, con mayor énfasis en el rigor que exige el acercamiento teórico y experimental a los fenómenos naturales y sociales acompañado de una disminución en los aspectos a veces meramente formales de las normas que establecen algunas agencias para citar la bibliografía o en el tipo de letra que debe utilizarse para reportar un escrito, pues estas normas no necesariamente son de aceptación universal en las revistas y publicaciones periódicas. La educación en niveles técnicos y tecnológicos es indispensable para que atienda muchas necesidades inmediatas, pero la creación de conocimiento, con contadas excepciones en Colombia, solamente se da en los espacios universitarios, particularmente en la formación doctoral. Una articulación real entre los diferentes niveles de formación, sin descalificar a unos o a otros, contribuirá con certeza a que mejore la calidad de vida de la sociedad en su conjunto.

Si bien sobre el sistema de educación superior recae la responsabilidad de impulsar la investigación en las diferentes áreas del saber y los campos de la innovación tecnológica, los demás actores distintos de la academia no pueden mirar de soslayo la importancia de contribuir a su consolidación. Las empresas, industrias y gremios en general, deben aportar recursos importantes a las actividades de investigación $e$ innovación pues no solamente se han beneficiado de alguna manera de los resultados del trabajo académico, sino que muchos de sus asociados se formaron en universidades públicas y tienen que retribuir algo de lo que recibieron. Si miran el presente y vislumbran un poco el futuro, deben acercarse a las universidades y solicitar propuestas de solución a los interrogantes sobre innovación y adecuación de tecnologías; con seguridad ahí encontrarán respuestas a varias necesidades y con investigadores colombianos solucionarán algunos problemas nacionales. En estas épocas de la Sociedad del Conocimiento está demostrado que es más acertado invertir en investigación e innovación que mantenerse al margen y emplear todos los recursos en importaciones. Como la investigación se aprende solamente con el ejercicio formal de investigar y esta actividad se realiza en Colombia mayormente en las universidades, particularmente en los programas de formación doctoral, es necesario impulsarlos y estimular su crecimiento en áreas y en número de estudiantes y graduados, sin detrimento de la calidad. El Estado debe adelantar políticas muy agresivas en esta materia, de manera que las universidades, cuya vocación y propósito sea la de adelantar programas de formación avanzada, reciban los recursos necesarios pero que a la vez se les exija la rendición de cuentas por el apoyo que se les dio.

De nada sirve tener listas nominales de grupos de investigación si los productos tales como publicaciones, patentes y expresiones artísticas no son visibles, particularmente por el impacto que tienen en el sector académico y en la sociedad en general. No puede descuidarse un producto tan importante como el número de estudiantes de programas de posgrado vinculados a los grupos y el número de los que culminan con éxito tales programas; este producto es ciertamente el indicador que debe tener mayor visibilidad. Hay que insistir en que lo más importante de una investigación no es la publicación de un artículo en sí misma, así se reciba un reconocimiento por pares y un estímulo económico, pues lo que se debe medir, como se mencionó, es el impacto de los resultados. Un ejemplo evidente es el caso de las patentes en las que las etapas intermedias no se hacen públicas; de ser así, no habría cómo preservar los derechos de autor o la propiedad intelectual. Sin embargo, hay que estimular las publicaciones pues es la manera de evaluar el conocimiento nuevo y de socializarlo, bien porque se corre la frontera del conocimiento, que es una de las funciones de las comunidades académicas, bien porque son la base de desarrollos e innovaciones tecnológicas que, en principio, deben beneficiar a la sociedad en su conjunto. Muchas veces se relaciona la investigación científica como actividad exclusiva de las ciencias naturales y las matemáticas y se olvida que la investigación en ciencias sociales no es solamente cuestión de opinión de una persona relativamente ilustrada. Sus formas rigurosas de aproximación ayudan a comprender e interpretar las realidades de nuestro entorno y es el sustento de las estrategias para, si es el caso, modificarlo.

Hay que estar preparados para enfrentar las tendencias mercantilistas que desean propiciar la competencia entre las instituciones educativas con prácticas propias del mundo de los negocios. En algunos sectores ha hecho carrera el concepto de que la educación no tiene 
impacto social sino beneficio individual del egresado, por lo que es más acertado financiar al estudiante mediante préstamos bancarios y disminuir los aportes del erario a la institución (10). Aun cuando en Colombia no se ha llegado a estos extremos ideológicos, debe mencionarse que en 2009 los rectores de las universidades públicas solicitaron un incremento en su presupuesto, por encima del contemplado en la ley 30 de 1992, que es inercial por estar atado exclusivamente al porcentaje de inflación. Pero los costos, particularmente de inversión, para responder con calidad a la demanda por educación superior aumentan en más puntos que el IPC. El Ministerio de Educación Nacional y el de Hacienda y Crédito Público asignaron recursos adicionales a la educación superior pero los giraron a entidades como ICETEX, cuyo objetivo principal es adjudicar créditos a los estudiantes que ya han sido admitidos en un programa que se ofrece en el país o en el extranjero.

La actitud y disposición del profesorado debe estar acorde con el fortalecimiento de las instituciones educativas y ser conscientes de la responsabilidad que tienen como formadores de ciudadanos libres, de personas críticas capaces de cuestionar y crear conocimiento. En el caso de los programas de pregrado, no se trata de que los estudiantes adelanten proyectos de investigación, pero la actitud investigativa debe estar presente en las actividades docentes. Para esto es necesario que el profesor sea un investigador activo, preferiblemente con título de doctor.

No hay que orientar esfuerzos exclusivamente para ir escalando posiciones en el ranking mundial, pues mientras en los indicadores se va mejorando, en los otros países también se mejora y quizás a una tasa mayor que la colombiana, como puede colegirse del análisis presentado por Brunner (11). Por ahora, con la interacción con comunidades académicas locales y particularmente extranjeras, mediante convenios y cooperaciones, se irá construyendo un verdadero sistema de calidad internacional y serán los pares y la sociedad quienes lo avalen sin que sea necesario que el reconocimiento esté amparado por la posición en un escala que, como se dijo, establece indicadores que no son en general comparables entre el hemisferio norte y el hemisferio sur.

\section{Referencias}

1. Perkin H. History of Universities, en P.G. Altbach (Ed.) International Higher Education. An Encyclopedia; New York and London: Garlang Publishing Inc.; 1991.

2. Le Goff J. La Civilización del Occidente Medieval, Ediciones Paidós Ibérica. Barcelona, España. 1999.

3. Fayad R. Educación Superior: De Servicio Público a Proyecto Cultural. Educación y Cultura. 1991;29-35.

4. Ministerio de Educación Nacional, SNIES, 24 de agosto; 2010.

5. Departamento Administrativo de Ciencia, Tecnología e Innovación (Colciencias). Informe ejecutivo. Convocatoria Nacional para la Medición de Grupos de Investigación, Tecnológica o de Innovación, 29 de agosto; 2010.

6. La investigación y su relación con la calidad de los Programas Académicos. En: http://www.ustatunja.edu.co/ciusta/images/ documentos/investigacion.pdf consultado en julio de 2010.

7. Ranking mundial de Universidades. En: http://www.webometrics.info/index_es.html consultado en septiembre de 2010.

8. Marginson S. Competition and Markets in Higher Education: a 'Glonacal' Analysis. Policy Futures in Education. 2004;2(2):175- 244.

9. Centro Interuniversitario de Desarrollo (CINDA). Educación Superior en Iberoamérica. Informe 2007.

10. Friedman, M and R. Free to Choose; Orlando, Fl: Harcourt, Inc., 1980.

11. Brunner, J. Mercados Universitarios: Los nuevos escenarios de la Educación Superior. Informe final del Proyecto FONDECYT No 1050138. 2007. 